\title{
Nonlinearity Engineering of Mode-locked Fiber Lasers: Similariton and Soliton- Similariton Lasers
}

\author{
F. Ömer Ilday ${ }^{1}$, Bülent Oktem ${ }^{2}$, Coşkun Ülgüdürr ${ }^{1}$ \\ ${ }^{1}$ Physics Department, Bilkent University, Çankaya, Ankara, 06800, Turkey, Ilday@bilkent.edu.tr \\ ${ }^{2}$ Material Science and Nanotechnology Graduate Program, Bilkent University, Çankaya, Ankara, 06800, Turkey
}

\begin{abstract}
Fiber lasers are attractive with their simplicity, high powers and low cost. However, propagation of short pulses in optical fiber leads to nonlinear effects, which limit the technical performance. These effects drive rich dynamics, which is interesting from a fundamental perspective. The nonlinear waves community has unraveled the fascinating world of solitons and similaritons through experiments in fibers. This paper overviews the recent development of the soliton-similariton laser. The original similariton laser was the first to work with nonlinear effects, rather than minimizing or compensating them. In the soliton-similariton laser, the propagation is strongly nonlinear everywhere.
\end{abstract}

\section{Introduction}

The physics of mode-locked fiber lasers displays a rich interplay of gain, dispersion and nonlinear effects. This is interesting in its own right since these lasers constitute a convenient experimental platform for studying nonlinear waves subject to periodic boundary conditions and dissipative effects, both of which profoundly alter the behavior of nonlinear waves. In addition to the well-known optical solitons [1], optical similaritons (self-similarly evolving pulses) have emerged as a new class of nonlinear waves in recent years [2]. Similaritons were observed in a laser cavity for the first time by Ilday, et al. [3]. These similaritons existed in segments of the cavity without any gain and loss, in order to avoid the large spectral broadening that is characteristic of amplifier similaritons. Formation of a self-consistent solution in a laser cavity requires the compensation of spectral broadening, which has proved to be non-trivial: Despite numerical predictions of their existence dating back almost a decade [4], amplifier similaritons had not been observed in a laser cavity to date.

Here, we review our recent experimental and theoretical work [5] on the discovery of a new mode-locking regime, where the pulse propagates self-similarly in the gain fiber with normal dispersion, and following spectral filtering, gradually evolves into a soliton in the rest of the cavity, where the dispersion is anomalous. In this laser, the distinctly different similariton and soliton pulses co-exist, demonstrating that transitions between these are possible; this is a new development, considering that all mode-locked lasers previously employed essentially a single type of a nonlinear wave propagation. We might speculate that other similar combinations should be possible in similar systems subject to feedback, potentially even outside of the field lasers and nonlinear optics, such as in electrical oscillators, fluid dynamics. Remarkably, this construct is robust against perturbations; although the pulse experiences nonlinear effects strong enough to cause unprecedented, order-of-magnitude variations of the spectral bandwidth, the laser exhibits excellent short and long-term stability.

\section{Results and Discussion}

The schematic of the experimental setup for the laser is illustrated in Fig. 1. Numerical simulations of the model laser, based on a modified complex Ginzburg-Lindau equation (CGLE), are used to analyze its operation. Parameters are chosen to match the experimental values. The evolution is illustrated by plots of the pulse duration and spectral bandwidth as functions of position in the cavity (Fig. 2a). The gain fiber has normal group-velocity dispersion (GVD), where the incident pulse evolves into an amplifier similariton. A bandpass filter then filters the spectrum. Following the filter, the pulse enters a long segment of single-mode fiber (SMF) with anomalous GVD, where it evolves into a soliton in the long SMF segment. The pulse undergoes soliton compression before its temporal and spectral widths stabilize. Similaritons have parabolic temporal profiles with linear chirp, and their temporal as well as spectral widths grow exponentially. In contrast, the first-order soliton pulse has a hyperbolic secant temporal profile and maintains a constant shape both in time and frequency, balancing nonlinear effects with dispersion. The transition from similariton to soliton is initiated by the bandpass filter, which filters both in the time and frequency domains due to the large chirp present. 
When the soliton re-enters the gain medium, it is shaped back into a similariton, which is an attractor state for any input pulse shape.

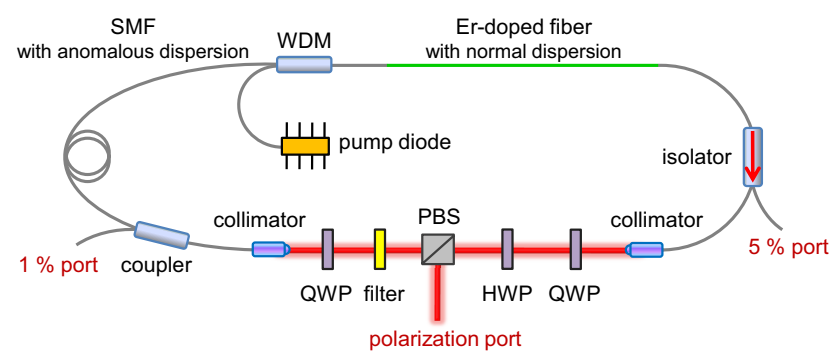

Fig. 1 The simplified schematic of the Er-doped fibre laser is shown. QWP: quarter-waveplate; HWP: halfwaveplate; PBS: polarizing beam splitter; WDM: wavelength-division multiplexer; SMF: single-mode fiber.
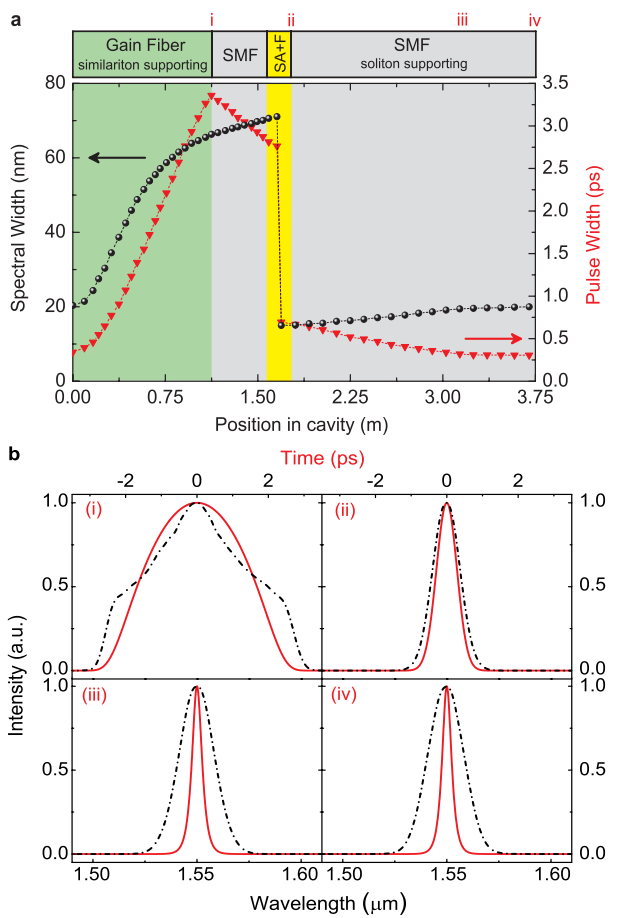

Fig. 2 (a) Conceptual model of the laser is shown with snapshot points indicated: (i) end of the gain fiber, (ii) after the filter, (iii) inside the SMF, (iv) entrance of the gain fiber. SA $+\mathrm{F}$ denotes the saturable absorber and the optical bandpass filter. Evolution of the spectral width (FWHM, black circles) and the pulse width (FWHM, red triangles) is plotted along the cavity. The shaded regions correspond to the main sections of the conceptual model. (b) Snapshots of the temporal (red, solid lines) and spectral (black, dashdotted lines) profiles of the pulse at the indicated locations.

Motivated by the simulation results, we constructed an Er-doped fiber laser. Characterization results for the laser operating with a $12 \mathrm{~nm}$-wide filter and total dispersion of $0.0136 \mathrm{ps}^{2}$ are shown in Fig. 3. We measured $12 \mathrm{~nm}, 64 \mathrm{~nm}$, and $85 \mathrm{~nm}$ of full-width at half-maximum (FWHM) values for the optical spectra from the 1\%, 5\%, and polarization rejection ports, respectively (Figs. 3a, b). The corresponding spectral broadening ratio is 7.1 . Figures $3 \mathrm{a}$ and $3 \mathrm{~b}$ show a good match between the simulations and the experiments. Pulse shapes are inferred from autocorrelation and spectrum measurements using the PICASO algorithm. The pulse shapes agree well with numerical simulations and match a parabolic (hyperbolic secant) temporal profile for the similariton (soliton-like) pulses shortly after the end of the gain fiber (near the end of the SMF section) (Fig. 3c,d). The laser generates $~ 750$-fs-long chirped pulses from the NPE port, which are compressed to $110 \mathrm{fs}$ with a $1.2-\mathrm{m}$-long $(-0.03 \mathrm{ps} 2$ of dispersion) SMF fiber outside the laser cavity. The zero-phase Fourier-transform calculation yields a theoretical lower limit of $75 \mathrm{fs}$ as shown in inset of Fig. 3f. The uncompressed FWHM widths of the pulses from the $5 \%$ and $1 \%$ ports are 0.82 ps (assuming parabolic shape) and 0.28 ps (assuming $\operatorname{sech}^{2}(\mathrm{t})$ shape), respectively (Fig. 3e, f). The laser is very stable both in the short and long terms: RF 
spectrum shows $105 \mathrm{~dB}$ (>120 dB, limited by the measurement) suppression of noise, including (excluding) the sidebands at 50 and $100 \mathrm{~Hz}$ coupled from the power supply (inset of Fig. 3e). The laser maintains uninterrupted modelocked operation for many weeks.
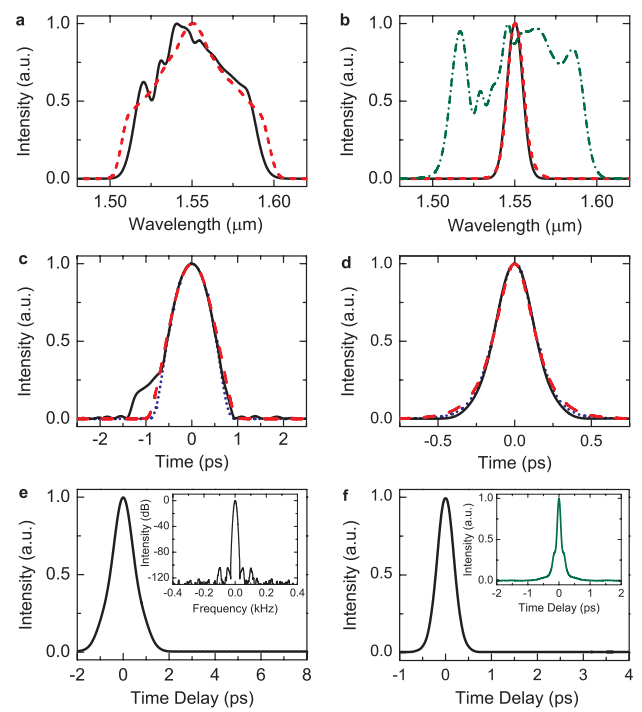

Fig. 3 Measured (black solid curve) and corresponding numerically simulated (red dashed curve) spectra of the pulse from (a) 5\% port, (b) 1\% port; measured spectrum (green dash-dotted curve) of the pulse from NPE rejection port are also plotted to show the spectral breathing. PICASO retrieved (black solid curve) and numerically simulated temporal intensity profile (red dashed curve) of the pulse from (c) $5 \%$ port with a parabolic fit (blue dotted curve) and (d) $1 \%$ port with a $\operatorname{sech}^{2}(\mathrm{t})$ fit (blue dotted curve). Intensity autocorrelation of the pulse from (e) $5 \%$ port, (f) $1 \%$ port, and NPE rejection port (f, inset). RF spectrum of the repetition of the laser with central frequency shifted to zero for clarity (e, inset).

Long-term power stability was characterized by sampling the optical power output of the laser at $1 \mathrm{~s}$ intervals up to $10,000 \mathrm{~s}$ (upper panel of Fig. 4). It is found that the root-mean-square (RMS) variations in the power output are within $0.033 \%$ over this range. Even though this level of stability is already good, it appears to be pump laser dominated and hence can be improved. Presently, the pump diode is operating at a constant current and the specified output power stability of the diode is $<0.5 \%$. Short-term power stability was characterized by measuring the relative intensity noise (RIN) (lower panel of Fig. 4). A high-dynamic-range and low-noise baseband signal analyzer (Rohde \& Schwarz, Audio Analyzer UPV) was used, following photodetection with a free-space InGaAs detector, and filtering off frequencies higher than $1.9 \mathrm{MHz}$. The integrated $\mathrm{RIN}$ is $0.008 \%(0.013 \%)$ over the frequency range $1 \mathrm{kHz}$ to $250 \mathrm{kHz}$ ( $3 \mathrm{~Hz}$ to $250 \mathrm{kHz}$ ) for the laser operating at total dispersion of $+0.0136 \mathrm{ps}^{2}$.

The single-side band phase noise and timing jitter of the laser system was characterized using direct photodetection (12 GHz photodetector, ET-3500 from Electro-Optics Technology). The RF signal at $1.3 \mathrm{GHz}$ (corresponding to the $12^{\text {th }}$ harmonic of the repetition rate) was selected with a bandpass filter and characterized using a signal source analyzer (Rohde \& Schwarz FSUP26). The measured phase noise and the equipment-limited noise levels are shown in Fig. 5. The corresponding timing jitter is 15.9 fs $(27.3 \mathrm{fs})$ from $1 \mathrm{kHz}$ to $10 \mathrm{MHz}(20 \mathrm{MHz})$, where 20 $\mathrm{MHz}$ is the Nyquist limit. The measurement is clearly limited by the stability of the internal reference oscillator of the signal source analyzer as well as added timing jitter during photodetection.

\section{Conclusions}

In conclusion, we have developed a novel mode-locking regime of an Er-doped fiber laser, with similariton and soliton propagation in each half of the cavity [5]. The similaritons are of the amplifier type, which constitutes their first experimental observation inside a laser cavity and completes the earlier development of the similariton laser [3]. Indeed, we can interpret this mode of operation as a dissipative similariton, where the dissipation is viewed in a general sense of energy non-conservation and not necessarily only loss; in this way we may anticipate further links of this work with the wider class of nonlinear dynamics in non-Hamiltonian systems. The combination of an optical filter to undo the spectral broadening of the amplifier similariton and soliton formation to reshape the pulse into a chirp-free pulse, which can 
reseed the similariton formation, is the key step in overcoming the instabilities that prevented the experimental demonstration of an amplifier similariton laser for nearly a decade. The transitions between the similariton and solitonlike pulses are inherently interesting due to their vastly different characteristics and lead to variations of spectral width of the pulse by an order of magnitude, an unprecedented factor. In the limit of increasing filter bandwidth, the laser becomes identical to the dispersion-managed soliton laser. In the other extreme of vanishing SMF section, the cavity becomes identical to that of an all-normal-dispersion laser. Thus, this new mode-locking regime sits at a nexus of all four known regimes of operation. Finally, it is remarkable that despite the influence of these strong nonlinear effects the laser is easier to mode-lock and more robust compared to any Er-fiber laser incorporating NPE in our experience. The asymptotic attractive nature of the amplifier similariton may be the key to the increased robustness against perturbations and low-noise operation of the laser. Detailed noise and stability characterization of the laser indicates the high potential of this laser for low-noise applications, particularly for optical frequency metrology. In addition, we are working on the development of a semi-analytic theory of the soliton-similariton laser.
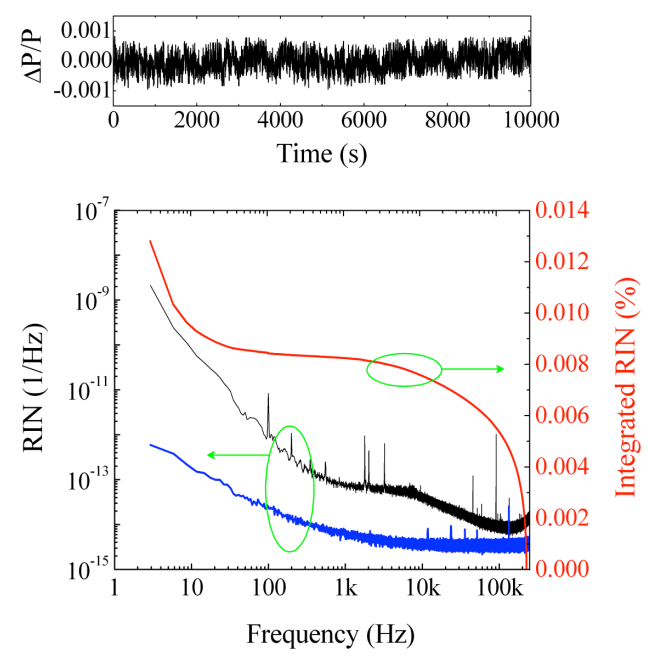

Fig. 4 Upper panel: Output power fluctuations measured at $1 \mathrm{~s}$ intervals up to 10,000 s. The RMS power drift level is $0.033 \%$ over $10,000 \mathrm{~s}$. Lower panel: Measured relative intensity noise (RIN) of the laser (black line) operating at total dispersion of $0.0136 \mathrm{ps}^{2}$. Blue line shows the equipment noise floor. Red line shows the total noise as a function of frequency.

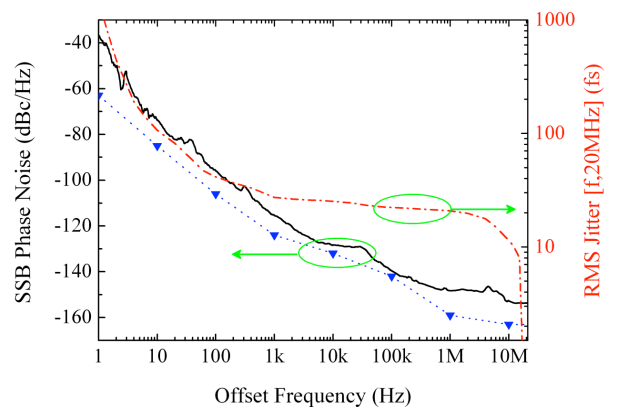

Fig. 5. Solid black line: single-sideband phase noise of the laser measured at $1.3 \mathrm{GHz}$. Dashed red line: RMS timing jitter obtained by integrating the phase noise. Dotted blue line: instrument noise limit.

\section{References}

1. Y. Kivshar, G. P. Agrawal, Optical Solitons: From Fibers to Photonic Crystals (Academic Press, San Diego, 2003).

2. J. M. Dudley, C. Finot, D. J. Richardson, and G. Millot, "Self-similarity in ultrafast nonlinear optics" Nature Phys. 3, 597-603 (2007).

3. F. Ö. Ilday, J. R. Buckley, W. G. Clark, and F. W. Wise, "Self-similar evolution of parabolic pulses in a laser." Phys. Rev. Lett. 92, 213902 (2004).

4. A. C. Peacock, et al., "Generation and interaction of parabolic pulses in high gain fiber amplifiers and oscillators," Conf. Opt. Fiber Commun. 2000, Tech. Dig., Paper WP4-1.

5. B. Oktem, C. Ülgüdür and F. Ö. Ilday, "Soliton-similariton fibre laser," Nature Photon. 4, 307, (2010). 